\title{
Adaptation of growth rate of Populus euramericana to light and nitrate proceeds via the vascular system
}

\author{
G.A. PIETERS and M.E. VAN DEN NOORT \\ Department of Plant Physiological Research, Wageningen Agricultural University, Gen. Foulkesweg 72, \\ $6703 \mathrm{BW}$ Wageningen, The Netherlands
}

Key words: absolute growth rate, adaptation, growing shoot, irradiance, nitrate, Populus euramericana 'Robusta', vascular system

\begin{abstract}
Shoots of Populus euramericana (Dode) Guinier cv. 'Robusta' were cultivated in subirrigated gravel culture at irradiances of 7.5 and $30.0 \mathrm{~W} \mathrm{~m}^{-2}$ and at 3 nitrate dosages in proportionality to irradiance. It is shown that (i) a poplar shoot adapts to a linear nitrate dosage in a similar way as to irradiance, viz. via adaptation of the volume of the growing shoot (GS), (ii) nitrate concentration of the organs, chlorophyll content, nitrate reductase activity of the leaves and shoot-root ratio are mainly a function of the ratio irradiance/nitrate-dosage (I/N-ratio) and (iii) the proposed morphogenic model provides a better basis for comprehension of these effects than the exponential model. It is suggested that the volume of GS is a reflection of the development of the vascular system. The constancy of the optimal I/N-ratio may point to a morphogenetic significance of protein synthesis for the enlargement of the vascular system.
\end{abstract}

\section{Introduction}

Ingestad (1982) gave a new impulse to the research on the relation between mineral nutrition and plant growth by designing the method of the relative addition rate coupled to the relative growth rate (RGR) of the whole plant to maintain a constant nitrate concentration. He also showed that in a young plant RGR and nitrate content are linearly correlated up to a certain optimum. The method of Ingestad is based on a model, assuming exponential growth. It appeared, however, that the period of exponential growth is short. Ingestad and Lund (1986) explain this by selfshading, although it has to be ascribed also to a declining ratio of growing and mature tissue. This model of growth has therefore a limited significance.

Pieters (1986) and Pieters and Van den Noort (1985) developed a morphogenic model of growth, in which each individual shoot, after a period of increasing absolute growth rate during adaptation to a given irradiance, continues to grow linearly with time. This model has a more general significance.

Preliminary results (Pieters and Van den Noort, 1985) indicated that sunflower and poplar adapt to a given, limiting and linear nitrate dosage in a similar way as to irradiance. Consequently, it must be possible to reach a steady state by growing shoots with a constant absolute growth rate, adapted to the limiting and linear nitrate dosage. While Ingestad adapts the nitrate dosage to the growth of the plants, in our experiments plants should adapt to the nitrate dosage. Pieters and Van den Noort (1985) found also that the nitrate requirement of a shoot is linearly related to irradiance (cf. Ingestad and Lund, 1986).

The morphogenic model of growth is based on an intensive study of the growth of individual leaves and internodes (Pieters and Van den Noort, 1988). It was established that these organs grow according to organ-specific patterns. 
A growth pattern can be defined as a 'fixed' relation between relative growth rate and age of the organ. If growth patterns are constant, differences in final size of e.g. leaves can only be explained by differences in the size of the initiated leaf primordia: primordia with equal size do not necessarily have an equal age.

Growth analysis also revealed that the initiation rate $(1 / \mathrm{P}$, leaves/day) of leaves with larger mature size $\left(\mathrm{L}_{\mathrm{m}}, \mathrm{cm}\right)$ was proportionally faster. At a temperature of $20^{\circ} \mathrm{C}$ this relation was: $1 / \mathbf{P}=\mathbf{a} \times \mathbf{L}_{\mathrm{m}}$, in which $\mathrm{a}$ is 0.042 leaves/ $($ day $\times \mathrm{cm})$. Consequently the plastochron $(P$, $=$ time unit between repetitive events) is inversely proportional to mature leaf length $\left(\mathrm{L}_{\mathrm{m}}\right)$. Leaf area production rate $(\mathrm{A})$ equals the production of one mature leaf per plastochron, thus:

$A=c \times$ leaf length $\times$ leaf width/plastochron, in which: $c=0.65$ (leaf area coefficient, Pieters and Van den Noort, 1985). Because also leaf width is proportional to $\mathrm{L}_{\mathrm{m}}, \mathrm{A}$ is proportional to $\mathbf{L}_{\mathbf{m}}^{3}$ or to a volume.

Since primordia are produced by the apex, we concluded that this volume might reflect the volume of the apex and measurement of the growing part of the primary stem (the growing shoot, GS) corroborated this (Pieters and Van den Noort, 1988). Mean RGR, calculated over GS, is a measure of the absolute growth rate of the average cell and remained constant throughout the whole growth period of the shoot. Differences in absolute growth rate of the shoot are due to the increase of the volume of GS. A larger apex produces proportionally larger primordia at a proportionally larger rate. In other experiments we found that diameter and length of GS increased linearly with time with a rate proportional to irradiance: the volume of GS is a power function of its linear dimensions and consequently total leaf and stem weight per shoot do not increase exponentially. When the increase of the size of GS stops, a shoot grows linearly with time. The increasing volume of GS was correlated with an increase of phyllotactic order.

There is a striking resemblance between the development of GS and that of the vascular system as described by Larson (1975, 1977, 1980). In a seed the vascular system contains two vascular bundles. During the development of the seedling the number of vascular bundles increases by systematic branching of the bundles to $3,5,8$ and 13 , while phyllotactic order increases to $1 / 3,2 / 5,3 / 8$ and $5 / 13$. At the same time the length and presumably also the diameter of each individual bundle increases proportionally. All this can fully explain the increase of the size of GS. The relation between structure and function is that bundles with a larger diameter produce larger primordia, while the correlated increase in the number of bundles increases leaf initiation rate. On the average leaf production rate per bundle appeared to be constant. It is generally accepted that growth vigour and phyllotactic order are correlated.

Larson (1975) also observed that Jong before a new primordium is visible at the apex, its primordial vascular bundle (procambial trace) branches off from the preceding mother trace deeper down under the apical dome and develops acropetally through the apical tissue. We may define the moment of initiation of a new leaf primordium as the moment of branching off of a new procambial trace. There are more observations, indicating that the development of the vascular system precedes the development of the shoot. Therefore we suggest that growth is a reflection of the development of the vascular system.

The aim of this report is to show that (i) the morphogenic model of growth is a better basis for understanding the effects of linear dosaging on plant properties than the exponential model; (ii) the adaptation of plant growth to a linear nitrate dosage proceeds via GS and (iii) the irradiance/nitrate-dosage is a determinant of deficiency symptoms.

\section{Methods}

Fresh cuttings (with low levels of reserves) of Populus euramericana (Dode) Guinier c.v. Robusta, on which one shoot was allowed to grow, were cultivated in growth rooms at $22^{\circ} \mathrm{C}$, $60 \%$ R.H. and a day length of $16 \mathrm{~h}$ on a nitratefree subirrigated gravel culture solution. Nitrate was added daily as a solution of $\mathrm{KNO}_{3}$, $\mathrm{Ca}\left(\mathrm{NO}_{3}\right)_{2}$ and $\mathrm{Mg}\left(\mathrm{NO}_{3}\right)_{2}$ (molar ratio: $35: 45: 20)$, according to the scheme in Table 1 . 
Table 1. Scheme of experimental treatments

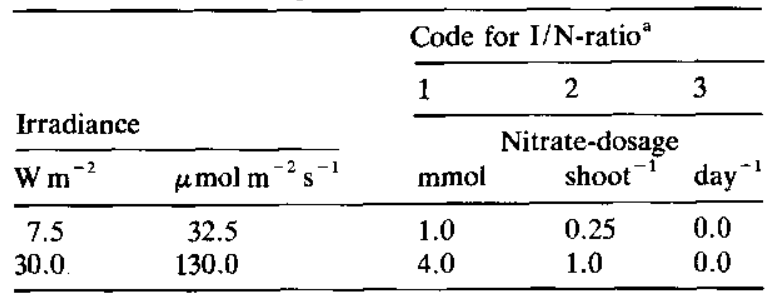

${ }^{a}$ Note that the ratio between irradiance and nitrate dosage is equal for both irradiances at each code.

The shoots were irradiated from above and from two sides. At the 2 highest nitrate dosages 6 shoots and at the lowest nitrate dosage 4 shoots per treatment were used. Three times per week leaf length and diameter and length of internodes were measured. The other measurements were performed on harvested shoots: two times at $0.0 \mathrm{mmol}$ nitrate and three times at the higher nitrate dosages.

Chlorophyll determination was performed after Bruinsma (1963), the chemical analyses after methods described by the Department of Soil Science and Plant Nutrition, Agricultural University, Wageningen.

\section{Results}

Although there were large differences in plant dry weight between the different treatments, relative growth rates at half mature length $\left(\mathrm{RGR}_{50}\right)$ of leaves and internodes (Table 2) were similar, except at a nitrate dosage of $0.0 \mathrm{mmol}^{\text {nitrate shoot }}{ }^{-1} \mathrm{day}^{-1}$ (code-3).

The amount of nitrate remaining in the nutrient solution just before the weekly change, appeared to diminish with the increasing growth rate of the plants. Adding up the weekly nitrate contents of the nutrient solution and total nitrate content of the harvested plants per treatment, recovery percentages between 95 and $106 \%$ were found at the two highest nitrate dosages (code 1 and 2). For plants grown in nitrate-free solution the recovery percentages were 97 and $57 \%$. This accentuates the importance of uniformity in the plant material at the start. Small differences in the thickness of a cutting lead to large differences in the ratio between the nitrate poor wood and the nitrate rich cortex tissue. Because we calculated an average nitrate concentration per $g$ dry weight of cutting, large errors resulted in the recovery percentage of plants which did not get any additional nitrate.

The development of the dimensions of the stems at different treatments in the course of time is shown in Figure 1. Each curve represents the form of the stem at a given measurement date; normally the measurements were done three times per week, as discernible from the figures. Each curve can be divided into a part with primary (GS) and a part with secondary growth, as indicated by the fitted line through measurement points, which are not shown. The higher irradiance and nitrate dosage, the higher was the rate of enlargement of GS. At the nitrate dosage of $0.0 \mathrm{mmol}$ nitrate shoot ${ }^{-1} \mathrm{day}^{-1}$ GS became smaller during the short period of growth before dormancy was induced.

The distribution of $\mathrm{N}_{\text {total }}$ concentration over the various organ groups (Fig. 2), chlorophyll content (Fig. 3) and nitrate reductase activity (NRA) of the leaves (Fig. 4) and shoot-root ratio (Fig. 5) are related to the $\mathrm{I} / \mathrm{N}$-ratio, as can be seen by comparing the right and the left side of the figures. A complication is that some plants at $7.5 \mathrm{~W} \mathrm{~m}^{-2}$ became dormant at limiting nitrate dosage.

Table 2. Mean plant dry weight in $\mathrm{g}$ and relative growth rates at half mature length $\mathrm{RGR}_{50}$ of leaves and internodes in $\%$ per day

\begin{tabular}{|c|c|c|c|c|c|c|}
\hline \multirow[b]{4}{*}{ Code } & \multicolumn{6}{|c|}{ Irradiance $\left(\mathrm{W} \mathrm{m}^{-2}\right)$} \\
\hline & 7.5 & 30.0 & 7.5 & 30.0 & 7.5 & 30.0 \\
\hline & & & RGR & & & \\
\hline & \multicolumn{2}{|c|}{ Dry weight } & Leav & & \multicolumn{2}{|c|}{ Internodes } \\
\hline 1 & 22.2 & 75.1 & 14.9 & 15.5 & 22.4 & 22.8 \\
\hline 2 & 17.0 & 54.9 & 14.9 & 15.9 & 22.1 & 21.9 \\
\hline 3 & 4.5 & 4.9 & 13.1 & 12.9 & 16.5 & 18.0 \\
\hline
\end{tabular}



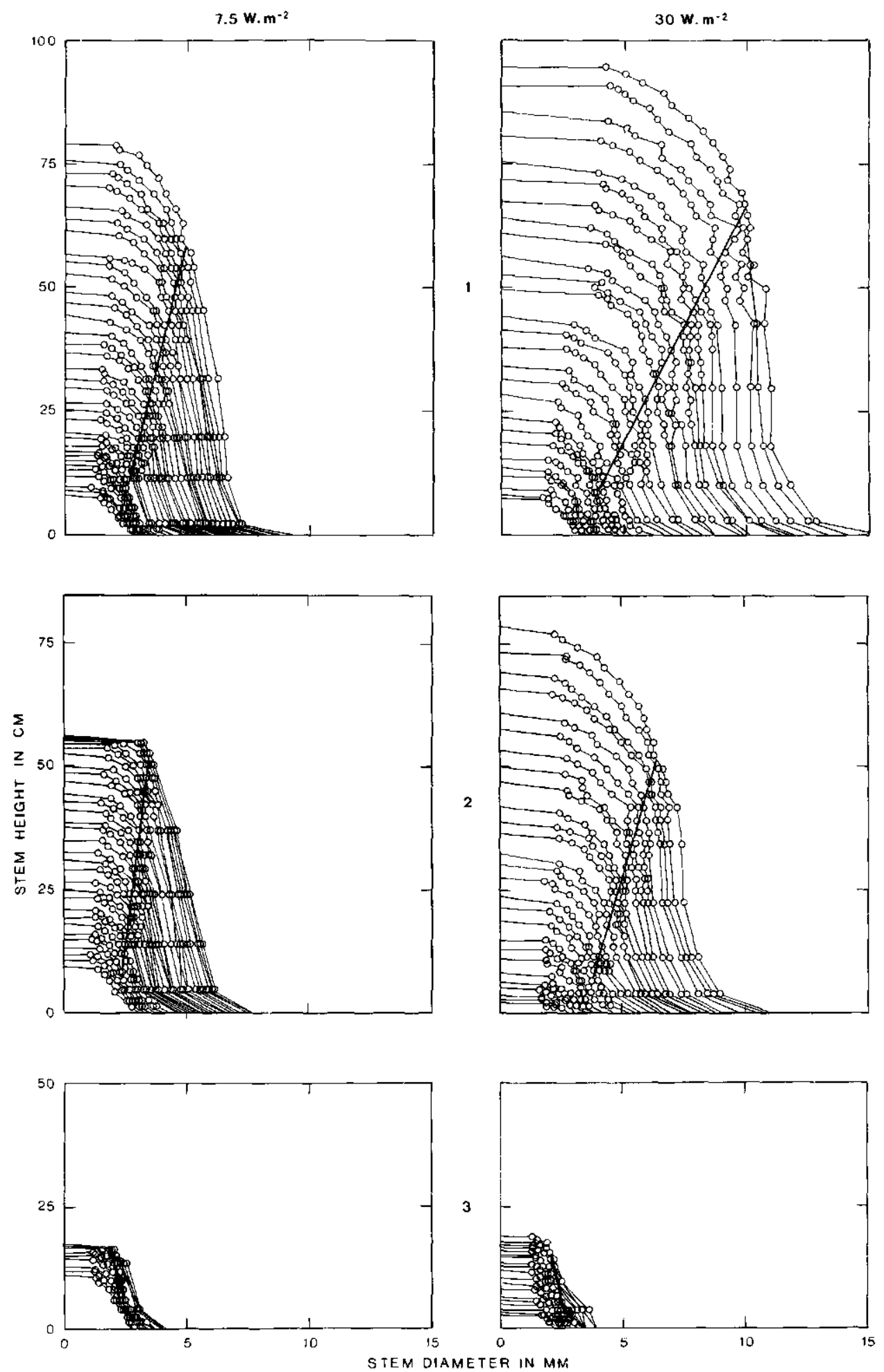

Fig. 1. The development of stem form in shoots from various treatments in the course of time. Each curve represents the mid-internode diameter versus cumulative internode length at a certain measurement date. The straight lines represent the base of the growing shoot (GS) and thus also the border between primary and secondary growth. Numbers between Figures indicate code for $\mathrm{I} / \mathrm{N}$ ratio. 


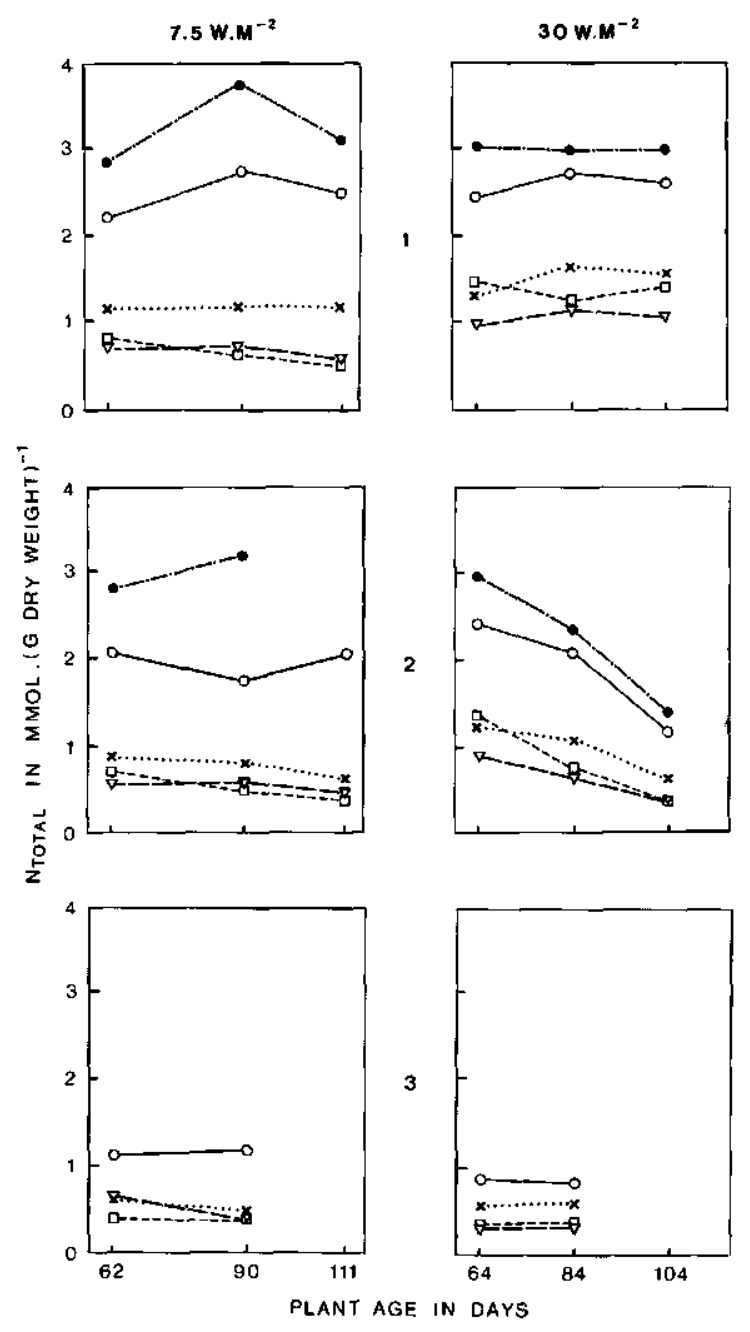

Fig. 2. Distribution of $\mathrm{N}_{\text {total }}$-concentration over organ groups of plants of the various treatments against shoot age. Legends: $O$ mature leaves; $\bigcirc$ growing leaves; $\nabla$ petioles; $\square$ stem; $\times$ roots. For numbers between Figures: See Figure 1.

Under conditions of strong nitrate deficiency the growing leaves tended to get a higher NRA than the mature leaves. The NRA of the roots is negligible.

The development of leaf weight is strongly related to the development of leaf area (Fig. 6). The slope of the correlation is a measure of leaf thickness and depended only on irradiance.

\section{Discussion}

The growth patterns of leaf length and width and internode length and diameter were independent

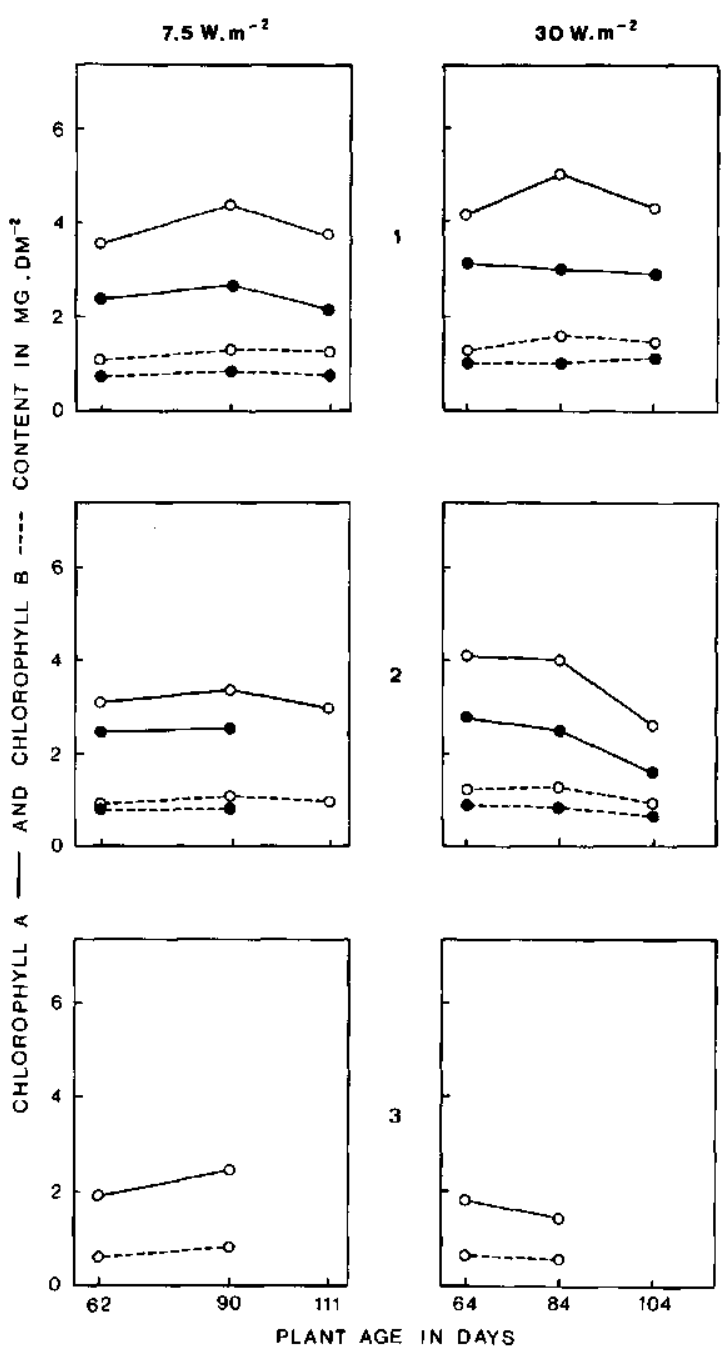

Fig. 3. Chlorophyll a and b content of growing (O) and mature $(O)$ leaves of plants of the various treatments against shoot age. For numbers between Figures: See Figure 1.

of irradiance and nitrate dosage (Table 2). In many experiments we observed that shoots, growing in constant conditions, maintained their growth patterns or became dormant. Shoots, cultivated at $0.0 \mathrm{mmol}$ nitrate shoot $^{-1}$ day $^{-1}$, however, were not able to maintain their growth patterns, presumably because the nitrate reserve in the cutting was used up before maturation and redistribution of nitrate could not sustain a high $\mathrm{RGR}_{50}$. The adaption of absolute growth rate to irradiance, nitrate- and presumably (Ingestad, 1987) phosphate-dosage thus proceeds mainly via the volume of the growing shoot (GS, Fig. 1), as expected in the morphogenic model. 


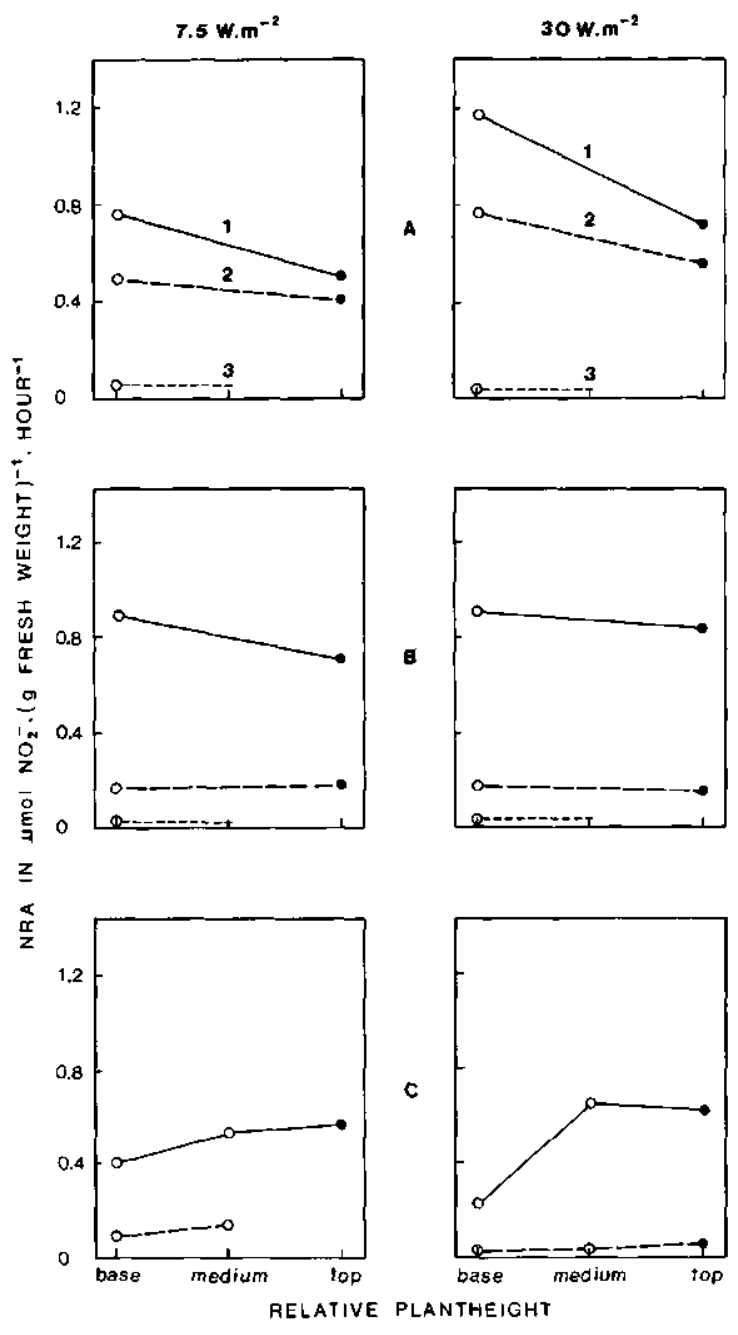

Fig. 4. Nitrate reductase activity (NRA) in growing (top, ๑), respectively young (medium, $O$ ) and old (base, $O$ ) mature leaves of plants of the various treatments. $A, B$ and $C$ indicate the first, second and third harvest.

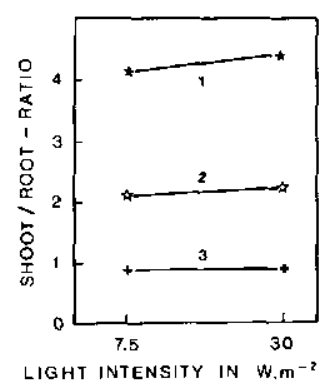

Fig. 5. The shoot-root ratio of plants of the various treatments was determined mainly by irradiance/nitrate ratio (I/N-ratio). Last harvest.

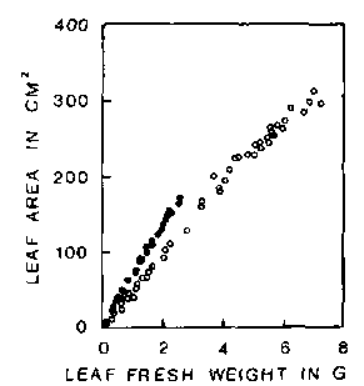

Fig. 6. The correlation between the development of leaf area and weight was only influenced by irradiance. 7.5 and $30 \mathrm{~W} \mathrm{~m}^{-2}$.

Because of the striking correspondence between the development of GS and the vascular system and e.g. the observation of Larson (1975) that vascular development precedes the initiation of a primordium, we suggest that the adaption of shoot growth is governed via an increase or decrease of the volume of the vascular system of GS.

While shoots are able to adapt to a limiting nitrate and presumably phosphate dosage, they cannot adapt to a deficiency of $\mathrm{K}$ - or $\mathrm{Mg}$-ions (Dorenstouter et al., 1985) without strong deficiency symptoms.

The question, whether shoots, growing at suboptimal nitrate dosage, can reach equilibrium, stop the expansion of the vascular system and attain linear growth, remains undecided. At code 2 and $7.5 \mathrm{~W} \mathrm{~m}^{-2}$ the shoots became dormant, while at $30 \mathrm{~W} \mathrm{~m}^{-2}$ the duration of the experiment was too short. As expected, also the shoots, grown at the highest nitrate dosage (code 1), did not reach linear growth, because an ever increasing illuminated leaf area (see Methods) leads to a continuous increase of GS within the duration of the experiment. Dry matter distribution and chemical composition and activity of the plants do not depend on the absolute irradiance or nitrate- (presumably also phosphate-) dosage, but on the ratio of these factors, as indicated by the code. In the present experiments the lowest I/N-ratio was the best and, in comparison to plants from other experiments growing without any nitrate limitation, the plants were growing nearly optimally. This means that there exists an optimal I/N-ratio. Although we did not yet analyze this ratio in respect to the resulting relative amounts of $\mathrm{C}$-, $\mathrm{N}$ - an P-compounds in the plant, 
we suggest that the constancy of the optimal ratio points to a morphogenetic significance of protein synthesis for the enlargement of the vascular system.

Linear dosaging of nitrate in relation to irradiance is an interesting alternative for studying the effects of ion nutrition on the growth of plants.

\section{Acknowledgement}

The members of the Department of Soil Science and Plant Nutrition of Agricultural University, Wageningen are duly acknowledged for their help and advice.

\section{References}

Bruinsma J 1963 The quantitative analysis of chlorophylls a and b in plant extracts. Photochem. Photobiol. 2, 241-249.

Dorenstouter H, Pieters G A and Findenegg G R 1985 Distribution of magnesium between chlorophyll and other photosynthetic functions in magnesium deficient 'sun-' and 'shade-' leaves of poplar. J. Plant Nutr. 8, 1089-1101.

Ingestad $T$ 1982. Relative addition rate and external concentration: Driving variables used in plant nutrition research. Plant Cell Environ. 5, 443-453.

Ingestad T and Lund A 1986 Theory and techniques for steady state mineral nutrition and growth of plants. Scand. J. For. Res. 1, 439-453.

Ingestad T 1987 New concepts on soil fertility and plant nutrition as illustrated by research on forest trees and stands. Geoderma 40, 237-252.

Larson P R 1975 Development and organization of the primary vascular system in Populus deltoides according to phyllotaxy. Am. J. Bot. 62, 1084-1099.

Larson P R 1977 Phyllotactic transitions in the vascular system of Populus deltoides Bartr. as determined by ${ }^{14} \mathrm{C}$ labeling. Planta 134, 241-249.

Larson P R 1980 Interrelations between phyllotaxis, leaf development and primary-secondary vascular transition in Populus deltoides. Ann. Bot. 46, 757-769.

Pieters G A 1986 Dimensions of the growing shoot and the absolute growth rate of a poplar shoot. Tree Physiol. 2, 283-288.

Pieters G A and Van den Noort M E 1985 Leaf area coefficient of some Populus euramericana strains. Photosynthetica 19, 189-193.

Pieters G A and Van den Noort M E 1988 Effect of irradiance and plant age on the dimensions of the growing shoot of poplar. Physiol. Plant. 74, 467-472. 